\title{
Preoperative Concern about Nausea and Vomiting and Postoperative Use of Antiemetics among Patients Undergoing Breast Cancer-Related Surgery
}

\author{
John L. Raytis ${ }^{1}$, Carolyn E. Behrendt ${ }^{2}$, Richard Obenchain ${ }^{3}$, Matthew Loscalzo ${ }^{3}$, Michael W. Lew ${ }^{1}$ \\ ${ }^{1}$ Department of Anesthesiology, City of Hope Comprehensive Cancer Center, Duarte, CA, USA \\ ${ }^{2}$ Information Sciences, City of Hope Comprehensive Cancer Center, Duarte, CA, USA \\ ${ }^{3}$ Patient and Family Services, City of Hope Comprehensive Cancer Center, Duarte, CA, USA \\ Email: jraytis@coh.org
}

How to cite this paper: Raytis, J.L., Behrendt, C.E., Obenchain, R., Loscalzo, M. and Lew, M.W. (2018) Preoperative Concern about Nausea and Vomiting and Postoperative Use of Antiemetics among Patients Undergoing Breast Cancer-Related Surgery. Open Journal of Anesthesiology, 8, 198-203.

https://doi.org/10.4236/ojanes.2018.86020

Received: May 17, 2018

Accepted: June 24, 2018

Published: June 29, 2018

Copyright $\odot 2018$ by authors and Scientific Research Publishing Inc. This work is licensed under the Creative Commons Attribution International License (CC BY 4.0).

http://creativecommons.org/licenses/by/4.0/

\begin{abstract}
Background: Postoperative nausea and vomiting (PONV) can lead to complications and increased healthcare costs. We investigated whether patient preoperative concern about PONV is associated with postoperative antiemetic use, independently of Apfel score. Methods: Patients eligible for study were English- or Spanish-speaking women with breast cancer undergoing mastectomy, lumpectomy or reconstructive surgery as outpatients during July 2014-July 2017, when the pre-anesthesia clinic routinely screened for preoperative concern via tablet computer-based survey. Excluded were patients who did not rate their concern or lacked Apfel score. Risk factors for concern were evaluated in a multinomial model adjusted for multiple hypotheses. Using generalized linear regression, preoperative concern was tested for association with number of antiemetics administered in the postanesthesia care unit. Results: Of preoperative surveys, 7.1\% (58/812) were excluded for missing data, leaving $\mathrm{n}=754$ surveys contributed by $\mathrm{n}=706$ subjects (age $26-80$ years). Patient preoperative concern ranged from none (32.8\%), mild (30.2\%), moderate $(22.9 \%)$, severe $(7.8 \%)$, to very severe $(6.2 \%)$. Adjusted for age, concern was increased by history of motion sickness (Odds Ratio 1.51, 95\% Confidence Interval 1.11 - 2.06) and history of PONV (9.02, $6.30-12.90)$ and decreased by prior surgery without PONV $(0.35,0.23-0.53)$ and Spanish as primary language $(0.42,0.25-0.68)$. Number of postoperative antiemetics, usually $1(41.2 \%)$ or $2(33.4 \%)$ drugs, was unassociated with preoperative concern before or after adjustment for Apfel score. Conclusions: Among women undergoing breast cancer-related surgery, preoperative concern about PONV varies by prior history of PONV and motion sickness and by ethnicity.
\end{abstract}


However, preoperative concern is not associated with postoperative antiemetic medications.

\section{Keywords}

Antiemetics, Patient Generated Data, Postoperative Nausea and Vomiting

\section{Introduction}

It is estimated that post-operative nausea and vomiting (PONV) affects one-third of surgical patients who undergo general anesthesia [1] and as many as 80 percent of high-risk patients [2]. PONV is an unpleasant experience that significantly decreases patient comfort and satisfaction [3]. In rare cases, PONV leads to serious medical complications, including dehydration, electrolyte imbalances, aspiration of gastric contents, esophageal rupture, suture dehiscence, and bleeding [4]. PONV can prolong recovery times and cause unintended hospital admissions [5]. It is not surprising, therefore, that PONV has a significant financial impact on the healthcare system-it is estimated that hundreds of millions of dollars are spent annually in the United States on PONV and its resulting complications [6].

There are several validated methods to determine a patient's risk for PONV. One of the most widely used, the Apfel score ranges between 0-4 with one point given for each of the following 4 factors: female gender, non-smoking status, prior PONV or motion sickness, and use of postoperative opioids [2]. The higher the patient's Apfel score, the higher the risk of PONV (score $0=9 \%$ risk, $1=$ $20 \%, 2=39 \%, 3=60 \%, 4=78 \%$ ) [2]. Although well-validated, the components of the Apfel score are largely inherent to the patient or procedure and not amenable to intervention. Patient concern about PONV, in contrast, might be possible to mitigate through intervention.

One method of ascertaining patient concern is via self-administered questionnaire using a tablet computer. Tablet-based screening provides multiple benefits, including effectiveness at collecting routine health information, general ease of use [7], and improved patient candidness [8]. However, some patients, including those with less than a high school education or multiple comorbidities, may have difficulty using such questionnaires [7].

Using data from a tablet-based survey in routine use at our institution, we undertook to assess patient preoperative concern about PONV in a high-risk population, namely female patients undergoing breast cancer-related surgery. Our aim was to learn whether a patient's concern about PONV is associated with her receipt of postoperative antiemetics. If an association exists, then an intervention to address preoperative concern may be developed and implemented with the goal of reducing the risk or severity of PONV and its impact on patient satisfaction, recovery, and healthcare costs. 


\section{Methods}

The study used a deidentified database of routinely generated data and was approved in advance by the hospital's Institutional Review Board, which granted a waiver of informed consent (IRB \#16044). Patients eligible for this study were English- or Spanish-speaking women with breast cancer undergoing mastectomy, lumpectomy or reconstructive surgery as outpatientsat City of Hope in Duarte, CA, during July 2014-July 2017. The period of study was chosen for practical reasons: during that time, the pre-anesthesia clinic routinely screened surgical patients using a tablet computer-based survey available in English and Spanish.

The survey collected, in addition to histories of PONV, motion sickness, and smoking, the patients' rating of their preoperative concern about PONV (using possible answers of None, Mild, Moderate, Severe, Very Severe, Don't Know, and Prefer Not to Answer). Survey responses became part of the electronic medical record, from which they and clinical and demographic characteristics were linked to data on postoperative use of opioids and antiemetics (ondansetron, promethazine, prochlorperazine, scopolamine, and metoclopramide) in the postanesthesia care unit. Apfel scores were calculated per published formula [2]. Once assembled, the database was stripped of personal identifying information.

Characteristics investigated for potential association with preoperative concern were history of PONV, history of motion sickness, current smoking, primary language, and age at survey. Those risk factors were evaluated in a multinomial model adjusted for multiple hypotheses using the Holm-Bonferroni method [9]. Preoperative concern was tested for association $(p<0.05)$ with number of postoperative antiemetics in a generalized linear regression model (normal distribution, $\log$ link), with and without adjustment for Apfel score. In all models, a generalized estimating equation with independent correlation was used to take within-patient correlation into account, for those subjects who underwent more than one surgical operation (each with its own preoperative survey) during the study period.

\section{Results}

Of operations with preoperative surveys, 7.1\% (58/812) were excluded for incomplete survey, leaving for study $n=754$ operations contributed by $n=706$ subjects (age 26 - 80 years, Table 1 ).

Of these subjects, 45 contributed 2 operations each, and 1 subject contributed 3 operations (mastectomy and 2 reconstruction procedures). Compared to the operations studied, those excluded for incomplete survey $(\mathrm{n}=58$ from $\mathrm{n}=57$ subjects) were statistically similar in age (mean $55.2 \pm 11.9$ vs $56.0 \pm 12.0$ years), current smoking ( $3.5 \%$ vs $4.2 \%$ ), diagnosis of invasive rather than in situ breast cancer (94.8\% vs $92.3 \%$ ), Apfel score (mean $2.91 \pm 0.65$ vs $3.06 \pm 0.75$ ), and number of postoperative antiemetics (mean $1.77 \pm 1.01$ vs $1.59 \pm 0.95$ ). Duration of operation, overall and by type of procedure, was also similar (data not 
Table 1. Multivariable model of preoperative concern about nausea and vomiting $(\mathrm{N}=754)$.

\begin{tabular}{|c|c|c|c|c|}
\hline Risk Factors & $\mathrm{N}(\%)$ & Odds Ratio & 95\% Confidence Limits & Holm p \\
\hline \multicolumn{5}{|l|}{ Prior PONV, By History of Major Surgery } \\
\hline Yes & $248(32.9)$ & 9.02 & $(6.30,12.90)$ & $<0.001$ \\
\hline No, Known to Have Had Surgery & $125(16.6)$ & 0.35 & $(0.23,0.53)$ & $<0.001$ \\
\hline No, Unknown Whether Had Surgery & $381(50.5)$ & 1.00 & & \\
\hline \multicolumn{5}{|l|}{ History of Motion Sickness } \\
\hline Yes & $264(35.0)$ & 1.51 & $(1.11,2.06)$ & 0.025 \\
\hline No & $490(65.0)$ & 1.00 & & \\
\hline \multicolumn{5}{|l|}{ Primary Language } \\
\hline Spanish & $57(7.6)$ & 0.42 & $(0.25,0.68)$ & 0.002 \\
\hline English & $697(92.4)$ & 1.00 & & \\
\hline \multicolumn{5}{|l|}{ Age } \\
\hline $26-69$ years & $641(85.0)$ & 1.00 & & \\
\hline $70-80$ years & $113(15.0)$ & 0.65 & $(0.43,0.96)$ & 0.062 \\
\hline \multicolumn{5}{|l|}{ Current smoker } \\
\hline Yes & $32(4.2)$ & * & & * \\
\hline No & $722(95.8)$ & & & \\
\hline
\end{tabular}

*Status as a current smoker did not improve the overall fit of the model to the observed data, hence was omitted from the final model. Holm adjustment, however, took smoking status into account as one of the multiple hypotheses tested.

shown). However, excluded operations were more likely to be mastectomy (67.2\% vs $43.0 \%)$ and less likely to be breast reconstruction only (10.3\% vs 27.5\%) (chi-square test, $\mathrm{p}<0.001$ ). Also, the patients with excluded operations were less likely to report a history of PONV (5.2\% vs $32.9 \%$ ) and more likely to speak Spanish as their primary language $(25.9 \%$ vs $7.6 \%)$ (both comparisons, Fisher's exact test, $\mathrm{p}<0.0001$ ).

Level of preoperative concern ranged from None (32.8\%), Mild (30.2\%), Moderate (22.9\%), Severe (7.8\%), to Very Severe (6.2\%). Unlike English speakers, no Spanish-speaking respondent rated concern above Moderate. According to multivariable analysis (Table 1), level of concern was increased by history of motion sickness and history of PONV and decreased by prior surgery without PONV, Spanish as primary language, and age 70 or older. Current smoking was unassociated with preoperative concern.

Apfel score ranged from 1 (1.2\%), 2 (21.2\%), 3 (47.6\%), to 4 (30.0\%). Preoperative concern about PONV was higher with Apfel score of 4 (Odds Ratio 4.91, 95\% Confidence Interval (CI) $3.41-7.07$, unadjusted $\mathrm{p}<0.0001)$ or Apfel score of $3(1.47,1.05-2.06$, unadjusted $p=0.024)$ relative to an Apfel score of 1 or 2 (being statistically similar, the latter were combined for efficiency). Of note, accounting for history of PONV eliminated the above-mentioned association between preoperative concern and Apfel score (data not shown).

Number of postoperative antiemetics ranged from 0 (9.7\%), 1 (41.2\%), 2 (33.4\%), 3 (12.2\%), to 4 (3.1\%) drugs. Number of antiemetics was unassociated with level of preoperative concern, either before taking Apfel score into account $(p=0.48)$ or afterward $(p=0.84)$. However, number of postoperative antiemetics did increase with Apfel score. Specifically, relative to an Apfel score of 2 (associated with an estimated mean use of $1.37,95 \%$ CI 1.21 - 1.56, antiemetics), an 
Apfel score of 1 was associated with lower mean use $(0.56,0.25-1.26 ; \mathrm{p}=0.03)$, and an Apfel score of 3 - 4 was associated with higher mean use (1.67, 1.47 $1.91 ; \mathrm{p}=0.003)$ of antiemetics.

\section{Discussion}

According to our study, preoperative concern about PONV is not predictive of actual PONV as measured by use of postoperative antiemetics. This finding contrasts with a previous report that, among patients undergoing ambulatory hand surgery under general anesthesia, PONV measured using a visual analog scale is associated with high level of preoperative anxiety [10]. It is likely that concern about the possibility of experiencing PONV does not equate with the degree of emotional distress and physiological changes associated with high level of anxiety, helping to explain the difference in findings between these 2 studies.

Among our patients undergoing breast cancer-related surgery, most (67.2\%) reported at least mild concern about PONV. We studied a population at high risk of PONV (77.6\% with Apfel scores of 3 or 4). A previous study of a lower-risk patient population reported far lower concerns for nausea $(8.7 \%)$ and vomiting (5\%) [11]. The finding that our patients' concern was increased by their history of motion sickness and PONV and decreased by prior surgery without PONV suggests that individual patients gauged their risk of PONV accurately.

We found that Spanish-speaking patients-despite taking our tablet-based survey in Spanish-reported lower levels of preoperative concern about PONV than English speakers did. Spanish speaking patients also had a lower rate of response to the survey question about preoperative concern. Taken together, these findings may indicate that less acculturated patients may be reluctant to disclose to providers views related to their healthcare that could be perceived as negative or critical. This interpretation is supported by a previous report that, compared to non-Hispanic whites, patients from ethnic and cultural minorities tend to underreport pain [12].

Limitations of the current study include its restriction to a single medical center and patient population. In addition, PONV among our subjects was not measured directly but was studied instead via the number of antiemetic medications administered postoperatively. Finally, Spanish-speaking patients were somewhat underrepresented in our sample, due to their higher rate of incomplete surveys. Whether those surveys were left unanswered for technical difficulty, which has been identified in other ethnic and cultural minorities [7], or from reluctance to report a negative viewpoint warrants investigation.

\section{Conclusion}

Among women undergoing breast cancer-related surgery, the level of preoperative concern about PONV varies by history of PONV and motion sickness and ethnicity. However, preoperative concern is not associated with use of postoper- 
ative antiemetic medications. From this finding, we conclude that intervention to address preoperative concern about PONV is unlikely to be effective in reducing actual PONV.

\section{References}

[1] Pierre, S. and Whelan, R. (2013) Nausea and Vomiting after Surgery. Continuing Education in Anaesthesia, Critical Care \& Pain, 13, 28-32. https://doi.org/10.1093/bjaceaccp/mks046

[2] Apfel, C.C., Läärä, E., Koivuranta, M., Greim, C.A. and Roewer, N. (1999) A Simplified Risk Score for Predicting Postoperative Nausea and Vomiting: Conclusions from Cross-Validations between Two Centers. Anesthesiology, 91, 693-700. https://doi.org/10.1097/00000542-199909000-00022

[3] Eberhart, L.H., Mauch, M., Morin, A.M., Wulf, H. and Geldner, G. (2002) Impact of a Multimodal Anti-Emetic Prophylaxis on Patient Satisfaction in High-Risk Patients for Postoperative Nausea and Vomiting. Anesthesia, 57, 1022-1027. https://doi.org/10.1046/j.1365-2044.2002.02822.x

[4] Apfel, C.C., Heidrich, F.M., Jukar-Rao, S., Jalota, L., Honruss, C., Whelan, R.P., Zhang, K. and Cakmakkaya, O.S. (2012) Evidence-Based Analysis of Risk Factors for Postoperative Nausea and Vomiting. British Journal of Anaesthesia, 109, 742-753. https://doi.org/10.1093/bja/aes276

[5] Buck 2nd, D.W., Mustoe, T.A. and Kim, J.Y. (2006) Postoperative nausea and Vomiting in plastic surgery. Seminars in Plastic Surgery, 20, 249-255. https://doi.org/10.1055/s-2006-951583

[6] Watcha, M.F. (2000) The Cost-Effective Management of Postoperative Nausea and Vomiting. Anesthesiology, 92, 931-933. https://doi.org/10.1097/00000542-200004000-00007

[7] Hess, R., Santucci, A., McTigue, K., Fischer, G. and Kapoor, W. (2008) Patient Difficulty Using Tablet Computers to Screen in Primary Care. Journal of General Internal Medicine, 23, 473-480. https://doi.org/10.1007/s11606-007-0500-1

[8] Feigelson, M.E. and Dwight, S.A. (2000) Can Asking Questions by Computer Improve the Candidness of Responding? A Meta-Analytic Perspective. Consulting Psychology Journal: Practice and Research, 52, 248-255. https://doi.org/10.1037/1061-4087.52.4.248

[9] Holm, S. (1979) A Simple Sequentially Rejective Multiple Test Procedure. Scandinavian Journal of Statistics, 6, 65-70.

[10] Roh, Y.H., Gong, H.S., Kim, J.H., Nam, K.P., Lee, Y.H. and Baek, G.H. (2014) Factors Associated with Postoperative Nausea and Vomiting in Patients Undergoing an Ambulatory Hand Surgery. Clinics in Orthopedic Surgery, 6, 273-278. https://doi.org/10.4055/cios.2014.6.3.273

[11] Moro, E.T., Godoy, R.C., Goulart, A.P., Muniz, L. and Modolo, N.S. (2009) Main Concerns of Patients regarding the Most Common Complications in the PostAnesthetic Care Unit. Revista Brasileira De Anestesiologia, 59, 720-724. https://doi.org/10.1016/S0034-7094(09)70096-0

[12] Mossey, J.M. (2011) Defining Racial and Ethnic Disparities in Pain Management. Clinical Orthopaedics and Related Research, 469, 1859-1870. https://doi.org/10.1007/s11999-011-1770-9 